\title{
Quantitative Detection of Low Energy Impact Damage in a Sandwich Composite Wing
}

\author{
Mark Seaver,* Engin Aktaş** and Stephen T. Trickey \\ Code 5673, Naval Research Laboratory, Washington, DC 20375, USA
}

\begin{abstract}
This work describes damage detection in a foam core composite wing $(1320 \mathrm{~mm} \times 152.4 \mathrm{~mm} \times 13.4 \mathrm{~mm})$ following a series of low energy impacts. Thirteen impacts (6-8 J deposited energy) were applied at adjacent locations approximately $1 / 4$ of the way out from the wing center. Following every one or two impacts, the wing was tested using static tip deflection and dynamic vibrational excitation. Static and dynamic strains were measured using eight fiber Bragg grating sensors. Dynamic acceleration was also monitored using three conventional accelerometers. The estimated bicoherence was used to detect the presence of damage-induced non-linearity in time-series data recorded from each sensor. Receiver operating characteristic (ROC) curves were constructed for each sensor based on 15 or more dynamic measurements made for each damage case. The ROC curves provide a quantitative, statistical approach to evaluating the damage detection capabilities of the various sensors.
\end{abstract}

Key Words: fiber bragg grating, structural health monitoring, impact damage, ROC curves, bicoherence, nonlinearity, sandwich composite, wing, probability of detection, probability of false alarms.

\section{INTRODUCTION}

Q UANTITATIVE comparisons of capabilities are a critical step in enabling informed decisions with respect to optimizing the many choices that face both researchers and owners in the field of structural health monitoring. Such comparisons allow the researcher to make informed choices with respect to what type of sensors, sensor locations, and analysis methods are best suited for monitoring the health of a particular structure. For an owner, combining knowledge of the probability of detection (POD) and the probability of false alarms (PFA) with costs of failure and repairs enables cost benefit analyses (Frangopol and Messervey, 2008), which can be used to choose detection thresholds or even determine whether or not a sensing network is economically viable. In spite of this importance, a search of the structural health monitoring (SHM) literature indicates that only a tiny fraction of the articles use terms indicating quantitative evaluation such as POD or receiver-operating characteristic (ROC) curves. An ISI Web of Science search using the descriptor 'structural health monitoring' returned 2853 results; adding 'probability of detection' to that descriptor returned just 14 results including four refereed journal articles (Lu and Michaels, 2005; Shook

*Author to whom correspondence should be addressed.

E-mail: seaver@nrl.navy.mil

**Present address: Civil Engineering Department, Izmir Institute of Technology, Izmir, Turkey.

Figures 2-11 appear in color online: http://jim.sagepub.com et al., 2008; Azarbayejani et al., 2008; Nichols et al., 2008). When the term 'receiver-operating characteristic' is added to 'structural health monitoring' only two refereed articles are found by the search engine (Milanese et al., 2008; Nichols et al., 2008).

Both ROC curves and POD analysis rely on multiple measurements to provide a statistical description of events and statistical descriptions are necessary when there is uncertainty in the measurement either from sensor noise or from structural influences. The main difference between the two approaches is that a ROC curve provides both the POD and the PFA as functions of the detection threshold in a single plot. ROC curves have a long history in signal processing in the Radar community (McDonough and Whalen, 1995; Olin and Meeker, 1996) and are extensively used in the medical community as well. (For a thorough description of how ROC curves work and their use and misuse in the medical world see Hopley and van Schalkwyk, 2007.) They are also used in the non-destructive evaluation community (Okure and Peshkin, 1995; Asraf and Gustafsson, 2003; Goebel et al., 2006) albeit sparingly. Their biggest benefit comes when combined with information as to the relative importance of true detections and false alarms. Then a ROC curve allows one to pick an appropriate decision threshold that can emphasize detections at the cost of more false alarms or visa versa (Swets, 1988). In the absence of information about the importance or costs associated with the possible outcomes, ROC curves allow for non-parametric comparisons between very 
different experimental variables such as sensor type, sensor location, and analysis methodologies. In this article, we demonstrate the production of ROC curves for detecting damage-induced non-linearity, use them to compare between fiber Bragg grating (FBG) strain sensors and conventional accelerometers, and finally evaluate the performance of the FBGs at different locations on the wing.

The structure under test was a full size replica of the composite wing from a small, unmanned aerial vehicle. The wing was damaged through a series of low energy $(\sim 7 \mathrm{~J})$ impacts placed at adjacent locations $\sim 1 / 4$ of the way out from the center of the wing. Both FBG strain gages and conventional accelerometers monitored the dynamic response of the wing to vibrational excitation. Our choice for a damage detection metric was the bicoherence function, a frequently used tool for detecting the presence of second-order (quadratic) non-linearities in time-series data (Hickey et al., 2008). Using non-linearity for damage detection is a well-known approach (e.g., Farrar et al., 2007) that offers the possibility of avoiding the need for baseline data from the undamaged (linear) structure, reduced sensitivity to ambient parameters such as temperature and loading, and it may obviate the need for broadband stochastic excitation (Nichols et al., 2005), although we use such excitation in this work.
The results from impacts 1-8 have been described previously with the conclusion being that while there is extensive damage to the foam core and limited amounts of subsurface skin damage, there was no evidence of damage using vibration-based features, either frequency response functions (FRFs) or bicoherence (Aktaş et al., 2009). This article extends that work through additional impacts and experimental modifications designed to increase the likelihood of detecting the damage as we seek to describe detection limits in terms of damage amount, sensor type, and sensor location for a chosen data analysis method (bicoherence). The experiment was stopped after impact 13 because it broke the skin, creating visible damage. At least 15 sets of dynamic data were recorded over multiple days at each damage level. The multiple data sets enabled a statistical evaluation of each sensor's ability to detect damage via ROC curves, which in turn allows evaluations of sensor type and location with respect to their damage detection performance.

\section{EXPERIMENTAL PROCEDURES}

The experiment has been described in detail previously (Aktaş et al., 2009). Figure 1(a) illustrates the mounting configuration for dynamic measurements, which was chosen because it approximates the way a

(a)
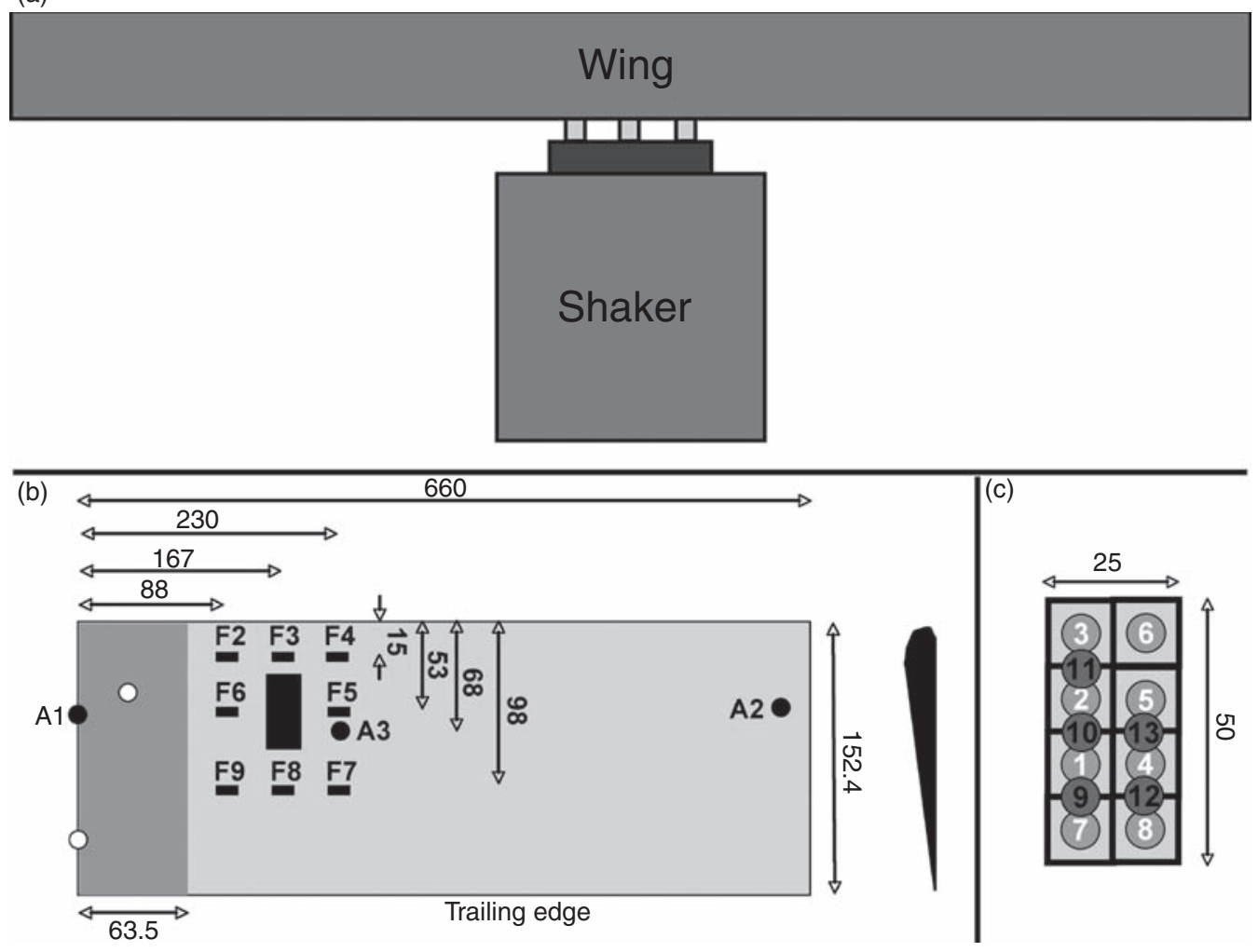

Figure 1. (a) A schematic showing the configuration for dynamic vibrational excitation, (b) a schematic of the damaged half of the wing illustrating the locations of damage (black rectangle), sensors (F2-F9 are FBGs and A1-A3 are accelerometers), and mounting points (white circles), (c) an illustration showing the location and order of the impacts within the damage area. The lengths are in millimeters. 
health monitoring system might be used on an aircraft. Figure 1(b) displays a schematic of half of the wing indicating the locations of sensors and damage. The other half was left untouched. The eight FBG strain gages are labeled F2-F9 and the three accelerometers are labeled A1-A3. Figure 1(c) displays the relative locations of the impacts within the damaged area. The wing, which has an airfoil shape with maximum dimensions of $1320 \mathrm{~mm} \times 152.4 \mathrm{~mm} \times 13.4 \mathrm{~mm}$, is a sandwich composite composed of four layers of carbon fiber fabric, surrounding an aluminum core that is connected to the fuselage, and a foam core for the rest of the wing span. The aluminum core occupies the central $127 \mathrm{~mm}$ of the wing and contains three holes in a triangle pattern for mounting to the fuselage. Differences between this work and the previous effort (Aktaşet al., 2009) include bolting the wing to an aluminum plate which in turn was mounted directly to the shaker platform rather than including an aluminum post, $51 \mathrm{~mm}$ long $\times 12.7 \mathrm{~mm}$ diameter. This change eliminates several peaks in the PSDs or FRFs, suggesting that some of the responses in the earlier work arose from side-to-side motion. It also concentrates the dynamic energy in the $38 \mathrm{~Hz}$ peak. Figure 2 illustrates these changes using the PSDs obtained from F5 after eight impacts in the two mounting configurations. All of the other FBGs and the accelerometers displayed similar changes. The $0-600 \mathrm{~Hz}$ frequency range contains all of the peaks below $926 \mathrm{~Hz}$, which was the Nyquist limit in the previous work. Note the factor of 5 increase for the $38 \mathrm{~Hz}$ peak. Also, the $220 \mathrm{~N}$ electrodynamic shaker with a $\pm 12.7 \mathrm{~mm}$ stroke used previously was replaced with a $500 \mathrm{~N}$ shaker with a $\pm 19.0 \mathrm{~mm}$ stroke to allow for higher amplitude excitation. While the maximum strain levels were essentially unchanged, the new shaker and mounting did provide a $30-40 \%$ increase in the RMS strain levels for $\mathrm{F} 6$, which records the largest signals. The larger shaker enabled the amplitude increases tested following impact 13, which will be discussed later.

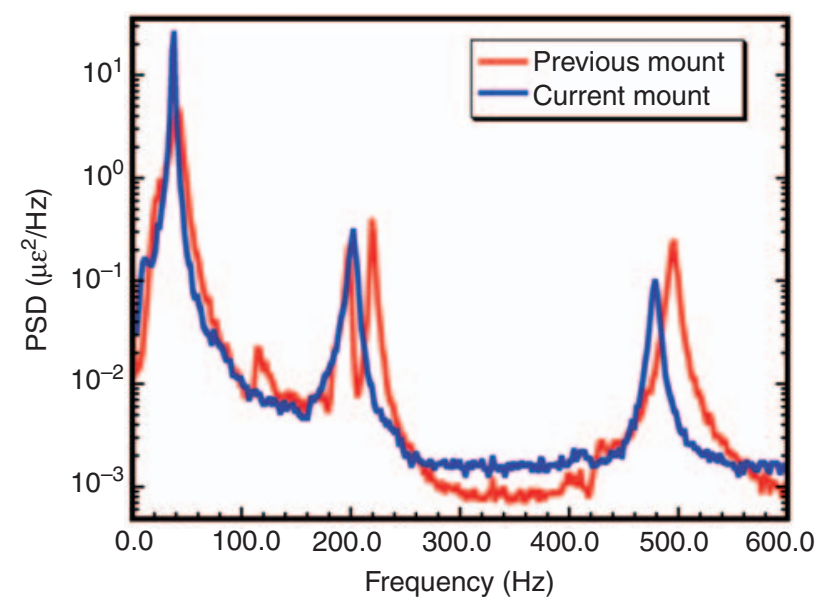

Figure 2. A PSD comparison for FBG 5 between the previous mounting configuration and the current mounting configuration.
The observed changes in vibrational dynamics and the increased excitation preclude direct comparisons with the previous paper (Aktaş et al., 2009). In addition FBGs 7, 8, and 9 were moved away from the trailing edge where maximum strains near $\pm 15 \mu \varepsilon$ were recorded to regions where the maximum recorded strains reached $\pm 40 \mu \varepsilon$. The final modifications, also designed to improve the likelihood of detecting changes in vibrational dynamics, were to double the recording time from 30 to $60 \mathrm{~s}$ and to increase the data rate from 1953 to $3906 \mathrm{~Hz}$. The recording time increase allows us to improve the signal/noise in the bicoherence calculation through increased averaging while retaining the bicoherence frequency resolution $(7.63 \mathrm{~Hz})$ through doubling the number of points used in the Fourier transform calculations that constitute the bicoherence.

Impact damage was created using a swing arm impactor with a $12.7 \mathrm{~mm}$ diameter hemispherical tup. Incident and rebound velocities were recorded providing measures of both incident and deposited energy for each impact. Deposited energies were kept between 6 and $7.5 \mathrm{~J}$. The impact locations covered a region roughly $25 \mathrm{~mm} \times 50 \mathrm{~mm}$, located about $150 \mathrm{~mm}$ from the center and $30 \mathrm{~mm}$ behind the leading edge of the wing. The initial eight impacts were spaced by $10 \mathrm{~mm}$ in a $2 \times 4$ grid resulting in minimal overlap between impact locations. For these experiments, the additional impacts were located between the centers of the initial impacts (Figure 1(c)), with the goal of creating lines of continuous damage in skin without any surface indications of the damage. In the previous work (Aktaş et al., 2009), NDE techniques showed that each impact left a $2-3 \mathrm{~mm}$ diameter region of damage in the skin and a $\sim 10 \mathrm{~mm}$ diameter region of crushed foam in the core. The idea was to 'connect the dots.' The thirteenth impact broke the skin creating clearly visible damage at which time we ended the experiment.

As we were unsure as to how or when the damage might produce detectable changes in the vibrational dynamics, two types of sensors were used to monitor the wing. Eight FBGs were mounted to the upper wing surface surrounding the damage area using a certified strain gauge adhesive. The spacing and location of these sensors was selected such that they were unlikely to be positioned directly above any structural damage, yet our model and previous experiments indicated that they were close enough to detect strain rearrangement that might occur due to local stiffness reductions. In addition, three conventional accelerometers were attached using tacky wax. The accelerometers were mounted at the wing center (A1), to monitor the excitation, near the wing tip (A2), for maximum accelerations, and near the damage (A3), looking for local changes and for comparison with one of FBGs. The accelerometer mounted nearest the damage was $15 \mathrm{~mm}$ away from F5, close enough to allow for a direct comparison of performance, 
and both sensors were about $50 \mathrm{~mm}$ from the nearest edge of the damaged region. The accelerometer outputs were low pass filtered at $1500 \mathrm{~Hz}$ to limit aliasing. Because the FBG demodulation system used in these measurements is non-linear, anti-alias filtering cannot be implemented without compromising the system's dynamic range (Todd et al., 2006). To minimize the potential for aliasing, we limit our analysis to $\leq 250 \mathrm{~Hz}$, which is well below the Nyquist limit of $1953 \mathrm{~Hz}$. A stochastic Gaussian vibrational excitation time series $(0-1500 \mathrm{~Hz})$ was calculated and used to drive the shaker. To accommodate the bandwidth of the Gaussian excitation, all sensors were sampled at $3906.25 \mathrm{~Hz}$. Following impact 13, different gain settings on the shaker amplifier were evaluated. These resulted in maximum strain amplitudes for F6 of $\pm 55 \mu \varepsilon, \pm 90 \mu \varepsilon$, $\pm 125 \mu \varepsilon$, and $\pm 170 \mu \varepsilon$. We will refer to these excitation levels subsequently as levels $1-4$, respectively. Following all other impacts, excitation level 1 was used.

One aspect of this effort was to relate dynamic damage detection capabilities to alternative damage indications. Thus, static strain measurements were made by mounting the wing in an apparatus that allowed us to apply a series of constant tip deflections $(0-38 \mathrm{~mm})$ to both wing tips (Figure 3). Only the FBGs could measure these static strains with repeated measurements showing a measurement reproducibility of $\leq 50 \mu \varepsilon$ due to quasistatic drift in the FBG demodulation hardware and $\pm 1 \mathrm{~mm}$ repeatability in the tip deflections.

The experimental procedure was to measure the static strains during tip deflections prior to and after each damage event. The initial damage states (1-9 impacts) were created using single impacts with the final two damage states using paired impacts so there were no static or dynamic measurements following impacts 10 or 12. Although impacts 1-8 were described previously, we repeated both the static and dynamic measurements on the wing with eight impacts prior to additional impacts. Thus, the data in this article start with impact 8 and continue through impact 13 .

In quantitative performance testing, the best practice is to make the measurements on individual test pieces each with 'identical' damage. In this case, with each wing costing $\$ 3000$, that approach would have been prohibitively expensive. Our alternative was to use the same wing but spread our measurements over three or more days. This insures that the damage is identical but removes manufacturing variability from the test matrix. To maximize measurement variability, we turned off all hardware after each day's measurements.

\section{DATA ANALYSIS}

Linear data analysis methods for damage detection are often complicated by the sensitivity of the structural response to changing ambient conditions (e.g., Sohn et al., 1999; Peeters and De Roeck, 2001). Previously, we have investigated the idea that non-linearity detection may avoid these complications by eliminating the need for baseline data from the healthy structure and minimizing the sensitivity to ambient conditions. One test, on a bolted joint, indicated that a non-linear metric called time-delayed transfer entropy could detect bolt loosening in the presence of $30^{\circ} \mathrm{C}$ temperature changes (Nichols et al., 2007). Subsequent investigations, suggested that the estimated bicoherence function, which is sensitive only to quadratic non-linearities, might provide similar or improved sensitivity with a significantly reduced computational effort.

\section{Bicoherence}

The bispectrum and bispectral analyses have been described by a number of authors (Brockett et al., 1988; Hinich and Wilson, 1990; Richardson and Hodgkiss, 1994; Rivola and White, 1998; Hillis et al., 2006; Petrunin and Gelman, 2008). The bispectrum is defined as the Fourier transform of the third moment about the mean. A linear system responding to a Gaussian input will, by definition, possess a zero bispectrum. However, a non-linear system responding to a Gaussian input will almost assuredly introduce thirdorder correlations between frequencies $f_{1}, f_{2}$, and $f_{1}+f_{2}$. The presence of such non-linearity will be easily

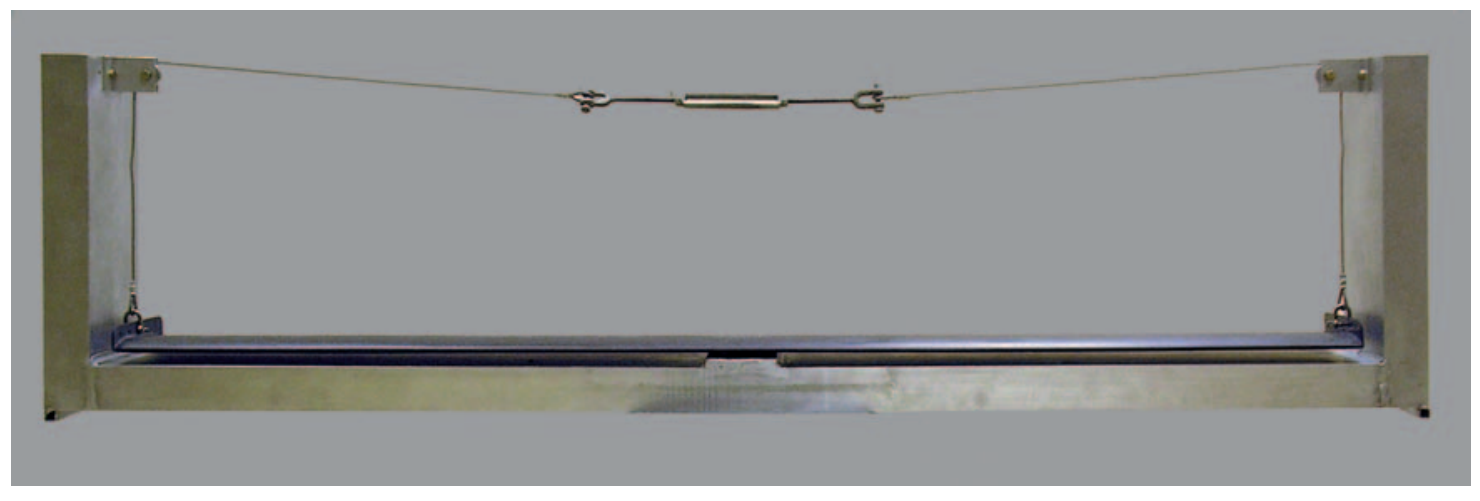

Figure 3. A photograph of the hardware used to impart wing tip deflections for static strain measurements. 
detected using the bispectrum. The metric is particularly useful in detecting the presence of quadratic nonlinearity but will not be sensitive to non-linear correlations lacking a quadratic component. While this limits its usefulness to specific types of damage, it seemed appropriate in this case where the damage was expected to include opening and closing of the skin/core disbond and possibly localized buckling of the skin. On the other hand, being limited specifically to quadratic nonlinearities may make it more sensitive than a general non-linearity detector when only quadratic effects are present. An intuitive way to think about the bispectrum is to consider it the frequency distribution of the skewness (Rivola and White, 1998).

Unfortunately, the bispectrum will also be non-zero for a linear system subject to non-Gaussian excitation (due to the presence of skew). To separate the influence of nonlinearity from a non-Gaussian input, the bispectrum needs to be normalized. This is typically done by dividing through by an appropriate triple product of the power density function of the response. Doing so removes all peaks in the bispectrum that arise due to a linear structure responding to a non-Gaussian input and results in a constant bicoherence function. Thus, frequency peaks in the bicoherence function clearly indicate non-linearity. There are two widely accepted approaches to this normalization. The signal processing literature normalizes with respect to the product $\left\{S_{x x}\left(f_{1}\right) S_{x x}\left(f_{2}\right) S_{x x}\left(f_{1}+f_{2}\right)\right\}^{0.5}$ where the $S_{x x}$ are single-sided autospectral density functions, $S_{x x}=X\left(f_{i}\right) X^{*}\left(f_{i}\right)$, and the $X^{\prime}$ 's are the Fourier transforms of the time series data. The result is a bicoherence function with the skewness as its magnitude. The engineering literature often uses the normalization described by Kim and Powers (1979), $\left\{\left|S_{x x}\left(f_{1}\right) S_{x x}\left(f_{2}\right)\right|^{2}\left|S_{x x}\left(f_{1}+f_{2}\right)\right|^{2}\right\}^{0.5}$, which produces a bicoherence $\leq 1$ that can be interpreted as the fraction of energy in the signal due to quadratic coupling. In this article, we follow the approach described in Richardson and Hodgkiss (1994) using the signal processing normalization. Thus our bicoherence function is calculated as:

$$
b_{x x x}\left(f_{1}, f_{2}\right)=B_{x x x}\left(f_{1}, f_{2}\right) /\left\{S_{x x}\left(f_{1}\right) S_{x x}\left(f_{2}\right) S_{x x}\left(f_{1}+f_{2}\right)\right\}^{0.5} \text {, }
$$

where $B_{x x x}\left(f_{1}, f_{2}\right)=\operatorname{re}\left\{X\left(f_{1}\right) X\left(f_{2}\right) X^{*}\left(f_{1}+f_{2}\right)\right\}$ is the unnormalized bispectrum and $X^{*}$ indicates the complex conjugate.

Convention suggests that a Fourier transform length that is the square root of the total number of points in the times series optimizes the trade off between frequency resolution and variance in a bicoherence calculation. This is supported by a recent study, which shows a broad maximum in the Fischer criterion versus relative segment length (Petrunin and Gelman, 2008). In this work, the total file length was $N=210 \mathrm{k}(\sim 60 \mathrm{~s}$ of data) and the FFT length was chosen as 512 points based on $N^{0.5}=460$. This FFT length provides a frequency resolution of $7.6 \mathrm{~Hz}$.

The bicoherence, as with any power spectral estimation technique, can be classified as either parametric or non-parametric as well as direct or indirect. In this case, because we are interested in any quadratic non-linearity and because we estimate the bicoherence from the data, the approach is direct and non-parametric. For damage detection, the direct approach offers major advantages in being an output only measurement and in allowing non-linearity evaluation using the variance calculated from the data. Estimating the variance from the signals avoids the need for baseline data from the undamaged structure provided the undamaged structure responds linearly to the excitation. The variance was calculated from the off diagonal elements of the bicoherence (Richardson and Hodgkiss, 1994) over the frequency interval 0-250 Hz using Equation (2).

$$
\operatorname{var}\left\{b_{x x x}\right\}=\left(1 / N_{0}\right) \sum\left|b_{x x x}\left(f_{1}, f_{2}\right)\right|^{2}
$$

Our non-linearity testing is based on hypothesis testing as described by Hinich and Wilson (1990). In this approach, the linear hypothesis is rejected when one or more peak heights exceed some threshold, $T_{a}$. In this case the threshold is calculated as:

$$
T_{\alpha}=\left(P_{\alpha} \operatorname{var}\left\{b_{x x x}\right\}\right)^{0.5},
$$

where the probability of $\left(\chi^{2}>P_{a}\right)=\alpha$ is derived from tables of the $\chi^{2}$ distribution with 2 degrees of freedom. The square root is taken because we want to compare peaks in the bicoherence (Equation (1)) with the threshold. It is necessary to multiply $P_{a}$ by the variance because our bicoherence data are not $\leq 1$. When using this approach the choice of $\alpha$ represents the probability of false alarms (Hinich and Wilson, 1990). For these data, we chose $a=95,99,99.5,99.9$, and 99.99 and used $P_{a}=2.995,4.605,5.300,6.910$, and 9.210, respectively. Note that the values of $P_{\alpha}$ are half the values given in tables of the chi-squared distribution. This reduction takes into account the fact that the variance contains equal amounts from both real and complex parts, which results in a $2 x$ overestimation when using Equation (2) since the $b_{x x x}$ contain only real values.

\section{RESULTS AND DISCUSSION}

The results from the first eight impacts have been described previously (Aktaş et al., 2009). At that stage, the damage was at best minimally detectable. Based on the thermographic evidence indicating isolated $2-3 \mathrm{~mm}$ diameter regions of internal damage in the skin, the additional impacts were placed between the previous impacts. The idea in these measurements was to create 
a continuous line of internal damage in the skin, which might lead to localized buckling and rearrangement of the strain field due to an extended reduction in stiffness in the wing skin. Thus, impacts 9, 10, and 11 were placed between the four previous impacts nearest the wing center (e.g., between 7 and 1, 1 and 2, and 2 and 3 in Figure 1(c)). Similarly, impacts 12-14 were to occur in parallel locations in the second row. Previous experience has shown that once significant core crushing has occurred, breakage of the skin occurs at lower impact energies. Thus, incident impact energies were reduced by about $20 \%$. This worked fine for impacts $9-12$, however, impact 13 broke the skin, resulting in an $\sim 10 \mathrm{~mm}$ diameter dimple about $2 \mathrm{~mm}$ deep showing broken fibers in the center.

Figure 4 shows the static strain measurement results for FBGs 2, 4, 6, and 8 following impacts 8, 9, 11, and 13. In this figure we see significant non-linearity in the response of FBG 8 for all four damage cases. For FBG 4 there is non-linearity for damage cases 11 and 13. FBG 2 only shows non-linearity for damage case 13 . FBG 6 shows slight reductions in strain for damage cases 11 and 13 but only at the largest strains. FBG 5 (not shown) also shows strain reduction, but only for damage case 13. FBGs 3, 7, and 9 show no evidence of strain rearrangement. The FBG 8 results indicate
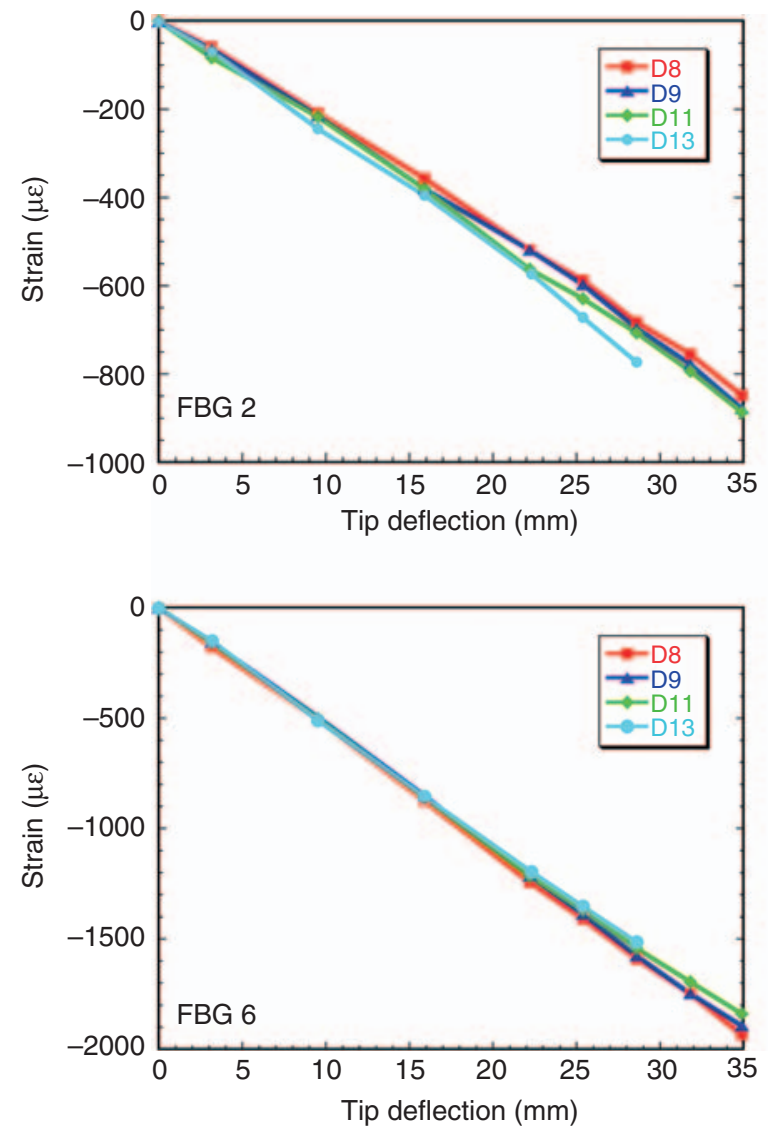

some non-linearity following impact 8 when strains exceed about $1500 \mu \varepsilon$ For impact 9, the non-linearity appears as the static strain exceeds about $1200 \mu \varepsilon$. Impacts 10 and 11 continue that trend, with the nonlinearity appearing at strains above $1000 \mu \varepsilon$. It is also clear at this level of damage that the strain field is rearranging to compensate for the damage as FBG 6 shows a slight reduction in strain at levels above of $1300 \mu \varepsilon$, eg. deflections greater than $25.4 \mathrm{~mm}$ ). Following impacts 12 and 13 the non-linearity seen by FBG 8 increased again with apparent changes as early as $400 \mu \varepsilon$. It became clear during the damage case 13 measurements that FBG 8 was chirping (displaying multiple peaks) at the $28 \mathrm{~mm}$ tip deflections. Thus measurements for this case were stopped at $28 \mathrm{~mm}$ of tip deflection. It's worth noting that for FBG 8 and damage case 13, the highest dynamic strain levels were $40 \mu \varepsilon, 65 \mu \varepsilon, 105 \mu \varepsilon$, and $115 \mu \varepsilon$ for excitation levels $1-4$, respectively.

The dynamic signals from all sensors were demeaned and normalized to unit variance prior to subsequent processing. The normalized signals were used to calculate the bicoherence, PSDs, and FRFs. To keep processing time reasonable, bicoherence estimates were calculated using 512 point FFTs with $50 \%$ overlap. This results in a frequency resolution of $7.6 \mathrm{~Hz}$. While we have not explored the effects of changing the FFT
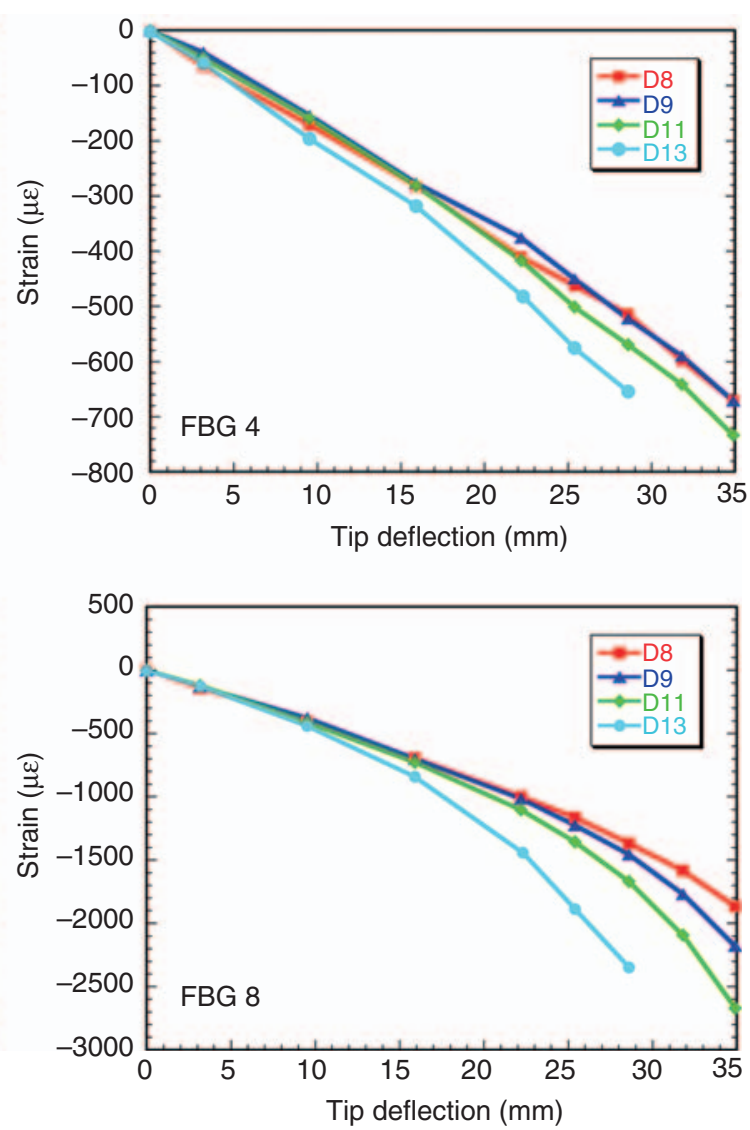

Figure 4. The static strain performance of the wing measured by four FBGs following impacts 8, 9, 11, and 13. 
length on detection sensitivity, the use of 512 point FFT corresponds with the rule of thumb for bicoherence calculations that suggests the optimum FFT length is the square root of the file length.

Figure 5(a) shows PSDs obtained from accelerometer 3 and FBG 5 for damage case 13 with level 1 excitation. (Note that these PSDs were calculated using 2048 point FFTs with $50 \%$ overlap, which results in resolution of $1.8 \mathrm{~Hz}$, not the $7.6 \mathrm{~Hz}$ resolution of the bicoherence calculations.) These two sensors were located $15 \mathrm{~mm}$ apart and $50 \mathrm{~mm}$ from the nearest edge of the damage area. Comparison between the two traces in Figure 5(a) shows how different the underlying response is when acceleration is compared to strain. While not shown, the reproducibility of the PSDs (or FRFs) was such that day-to-day differences were at least as large as any changes due to damage and/or excitation amplitude, with the peak near $200 \mathrm{~Hz}$ showing the most variability. In spite of the fact that the normalized noise (the PSD values approaching $2 \mathrm{kHz}$ ) for the accelerometer is
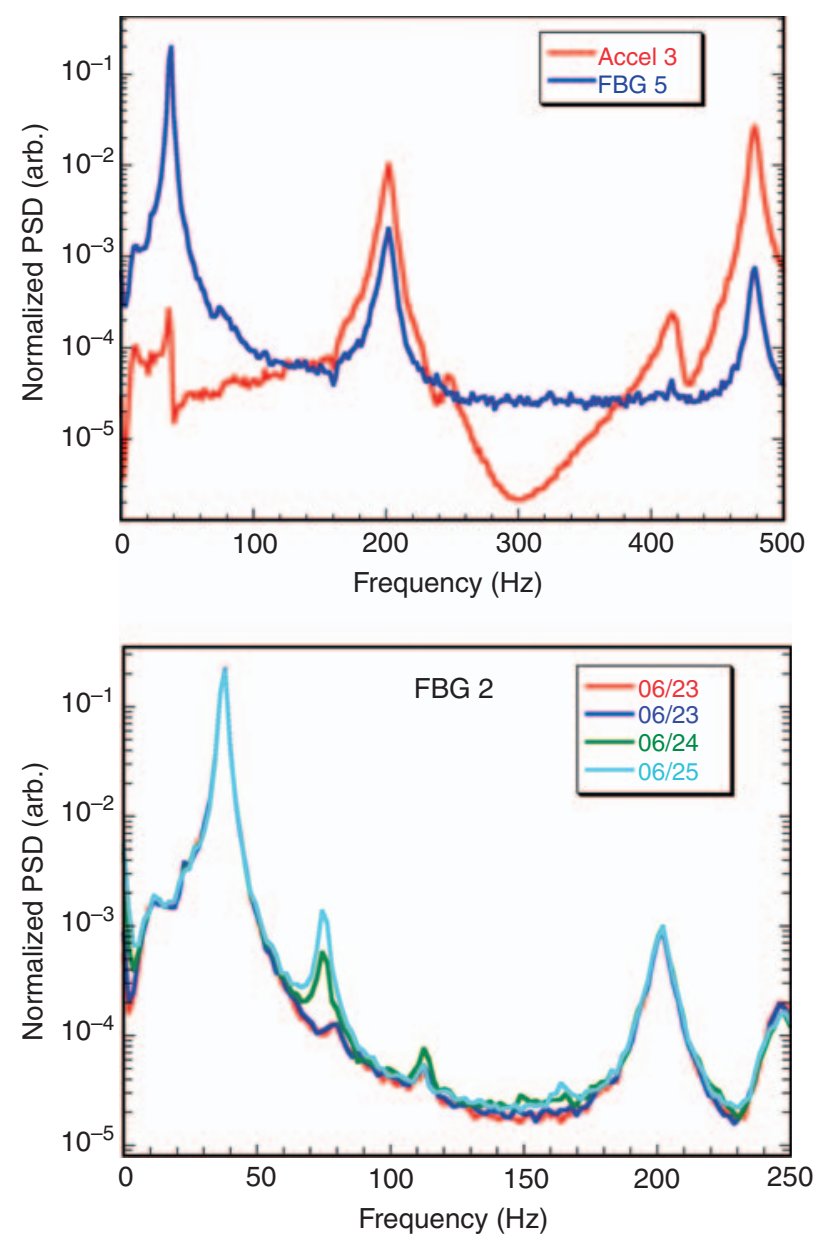

Figure 5. (a) A comparison of the PSD results for closely located sensors FBG 5 and accelerometer $A 3$ following impact 13 with level 1 excitation, (b) the variability in the PSDs for FBG 2 following impact 13 with level 4 excitation. Note the peaks near 78 and $112 \mathrm{~Hz}$, which are at the second and third harmonics of the $39 \mathrm{~Hz}$ fundamental. nearly two orders of magnitude lower than the normalized noise for the FBG, our analysis (described below) shows better damage detection with the FBGs. Figure 5 (b) displays the $0-250 \mathrm{~Hz}$ region of the PSD from four measurements of damage case 13 using level 4 excitation looking at FBG 2. In this plot, peaks appear near 76 and $114 \mathrm{~Hz}$, which are $2 x$ and $3 x$ the fundamental frequency making it a likely that these are harmonics and thus an indication of non-linearity. Figure 5(b) shows considerable inconsistency in the amplitude of both of these frequencies, which would make direct analysis difficult. As expected, reducing the excitation reduces the amplitudes of these two frequencies with the second harmonic $(114 \mathrm{~Hz})$ only seen occasionally for level 3 excitation and not at all for levels 2 and 1. The first harmonic $(76 \mathrm{~Hz})$ is seen consistently, but the amplitude is smaller than the smallest amplitude seen in Figure 5(b).

Figure 6 shows bicoherence contour plots from the sensor data shown in Figure 5(a). The top plot
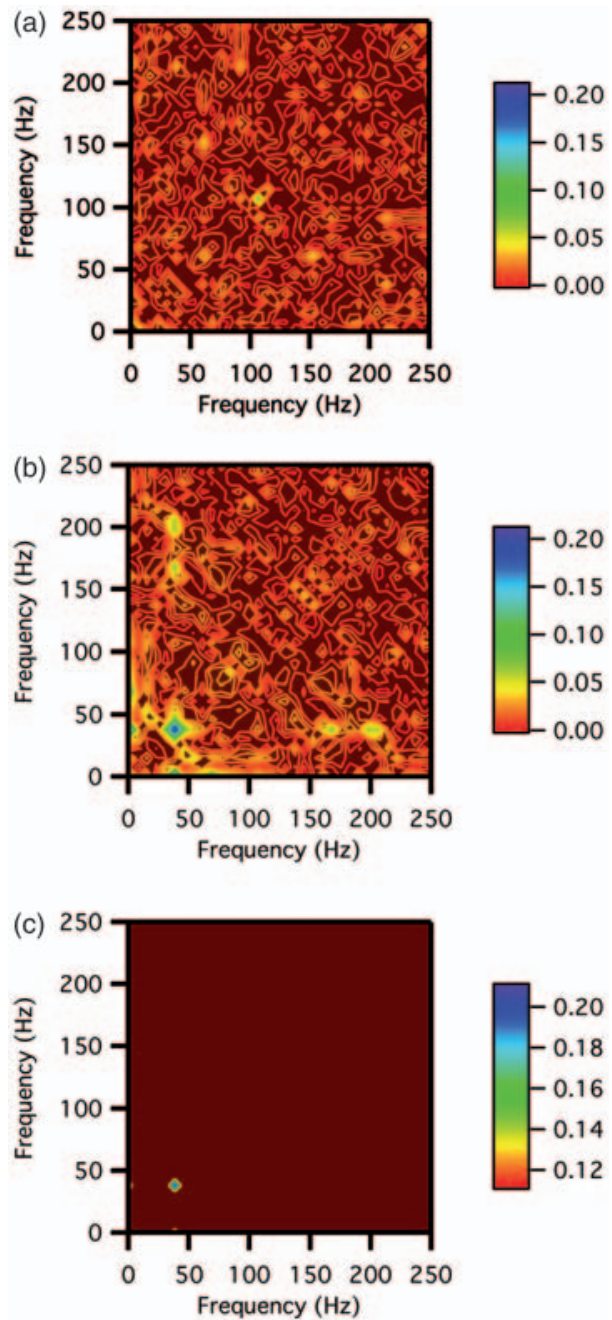

Figure 6. Bicoherence contour plots for: (a) accelerometer A3, (b) FBG 5, and (c) the thresholded plot for FBG 5. The damage level is 13 , the excitation level is 1 , and (a) and (b) use the same intensity scales. The threshold in (c) is set at the $99.9 \%$ confidence level. 

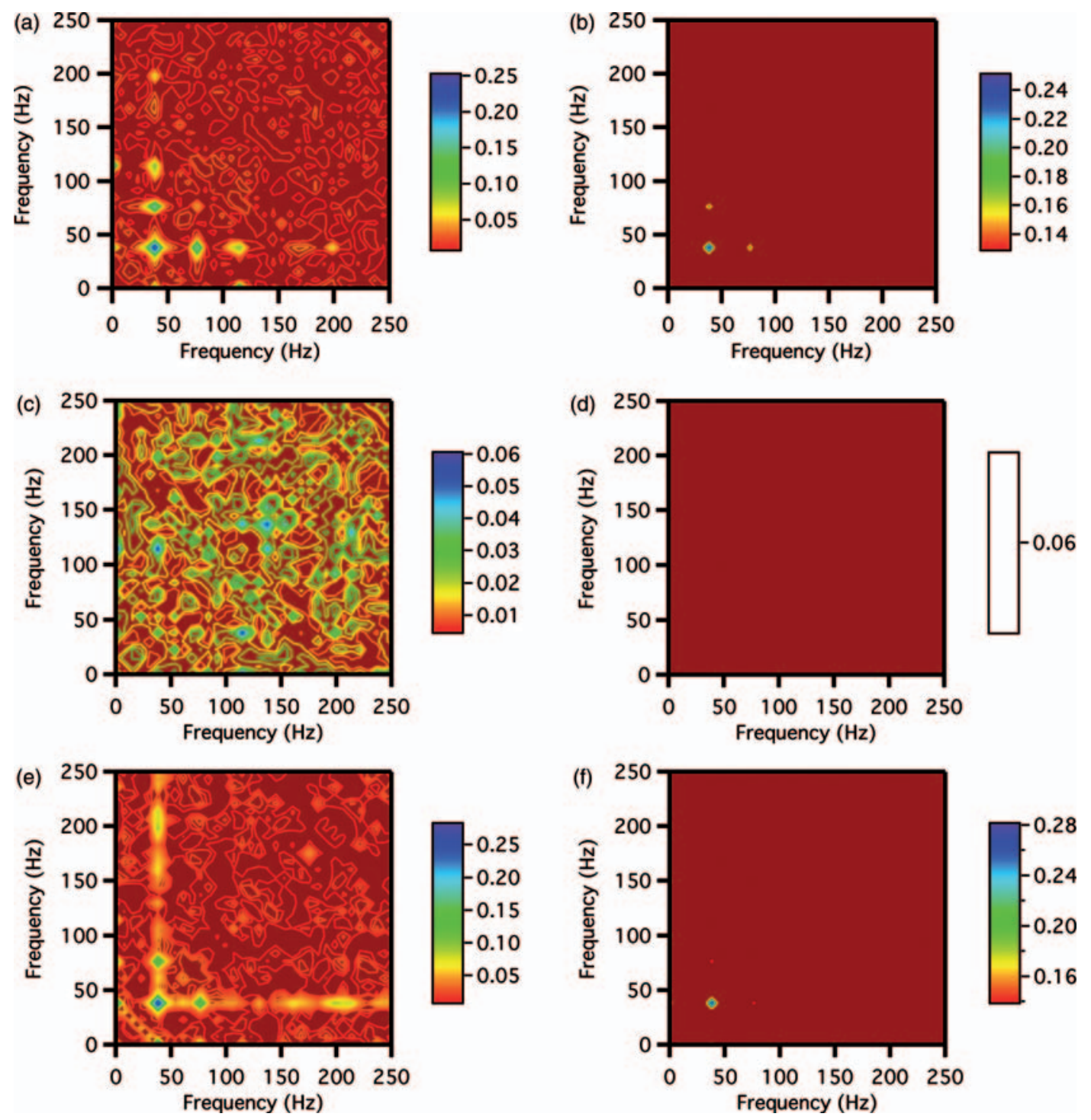

Figure 7. Bicoherence contour plots (left column) and thresholded bicoherence plots (right column) for FBGs 2 (a and b) and 7 (c-f). The results for FBG 7 illustrate the worst case variability between two consecutive measurements (c and e vs $d$ and f).

(Figure 6(a)), for accelerometer 3, shows only one small self-peak at $(110,110)$ with a maximum bicoherence of 0.055. The fact that this peak does not correspond to any of the wing's structural modes suggests that this is a noise peak. The middle plot (Figure 6(b)), for FBG 5, shows a strong self-peak at $(39,39)$ plus two smaller cross peaks at $(39,160)$ and $(39,200)$, all at structural modes. The FBG 5 self peak has an amplitude of 0.20 . Figure 6(c) (bottom) shows a thresholded bicoherence plot for FBG 5. In a thresholded plot, the minimum is set by the threshold as calculated using Equation (3) and data smaller than the threshold are ignored. The thresholded plot shows only one peak exceeding the $99.9 \%$ variance threshold $(0.112)$ for this measurement. Note that the variance changes from sensor-to-sensor and day-to-day. Based on our earlier discussion of POD and PFA, this is considered an indication of non-linearity for this sensor, damage, and excitation level. Meanwhile the fact that no peak in the accelerometer results exceeds the threshold (0.059) indicates linear behavior for that sensor.

Figure 7 illustrates additional aspects of these measurements. In this figure, the left hand column, (a, c, and e) shows unthresholded bicoherence results, while the right-hand column (b, d, and f) shows thresholded bicoherence plots for the same data. The data illustrate the differences seen between sensors (rows 1 and 2) and a worst-case example of differences between measurements for the same sensor (rows 2 and 3). Figure 7(a) and (b) are from FBG 2 with Figure 7(a) showing peaks at the $39 \mathrm{~Hz}$ fundamental, its first and second harmonics, and a small peak near $200 \mathrm{~Hz}$. Figure 7(b) shows that both the $39 \mathrm{~Hz}$ fundamental and it's first harmonic are non-linear above the $99.9 \%$ variance 

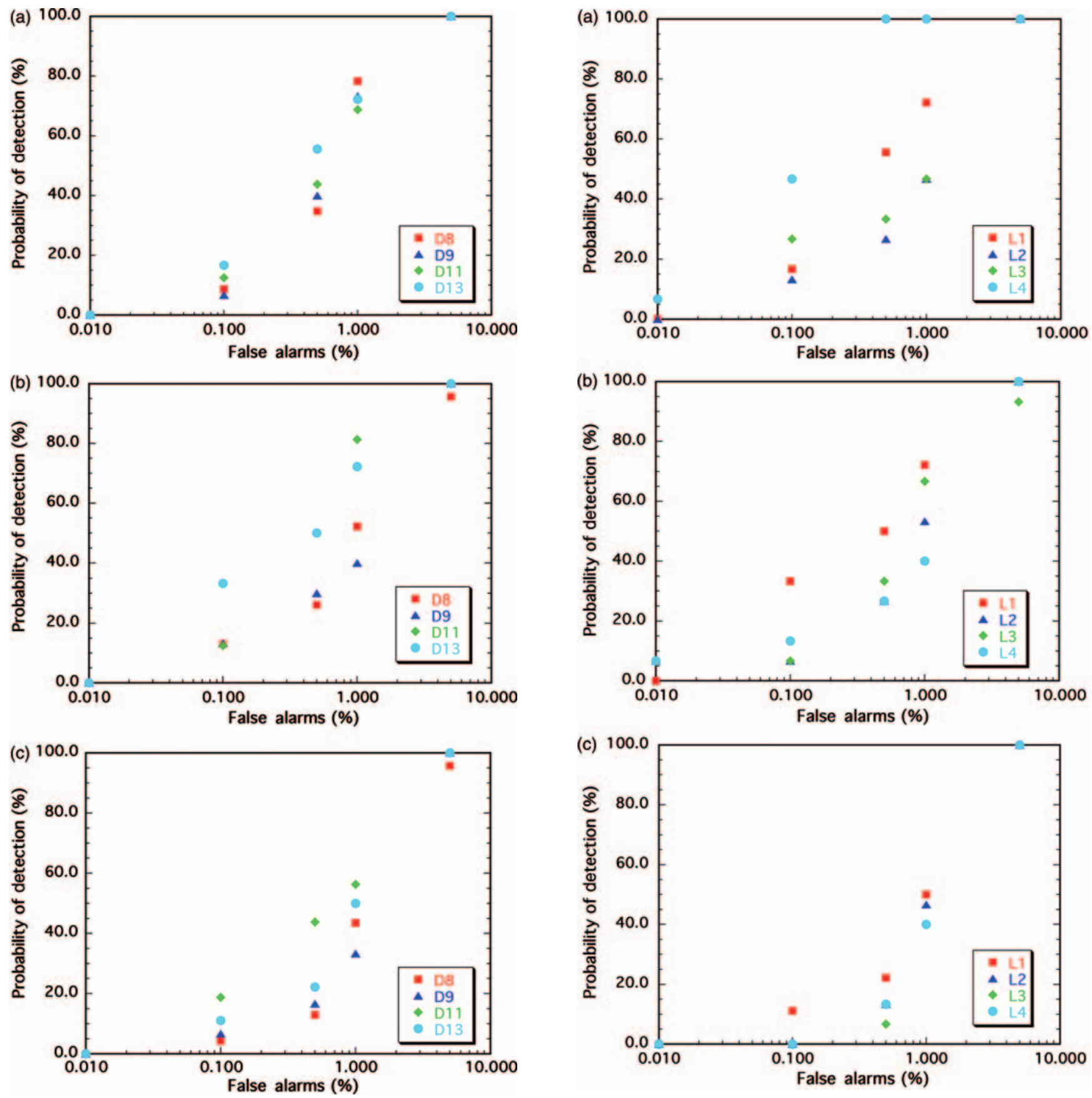

Figure 8. ROC curves for the accelerometers $A 1$ (a), $A 2$ (b), and A3 (c) as the level of damage increases from 8 to 13 impacts.

Figure 9. ROC curves for the accelerometers $A 1$ (a), A2 (b), and $A 3$ (c) for increasing excitation L1-L4 following 13 impacts.

threshold (0.134). In contrast, the results (from the same measurement) for FBG 7, Figure 7(c) and (d), show only small peaks in the bicoherence none of which exceed the $99.9 \%$ variance threshold (0.058) indicating no non-linearity at this location. When the measurement was repeated, about $3 \mathrm{~min}$ later, FBG 7 shows non-linearity in the $39 \mathrm{~Hz}$ peak, and in it's first harmonic $(99.9 \%$ threshold $=0.143)$. Note that the peak bicoherence amplitude for FBG 7 increased from $5.6 \times 10^{-2}$ to

$2.7 \times 10^{-1}$ for these consecutive measurements. This clearly shows that there are times where the variability in the non-linearity is large. At this time, it is unclear whether such variability is in the FBG demodulation hardware or if it illustrates the variability of the measurement.

Figures 8-11 display the ROC curves derived for all 11 sensors. In general, there is limited consistency in terms of expectations that increasing damage or 

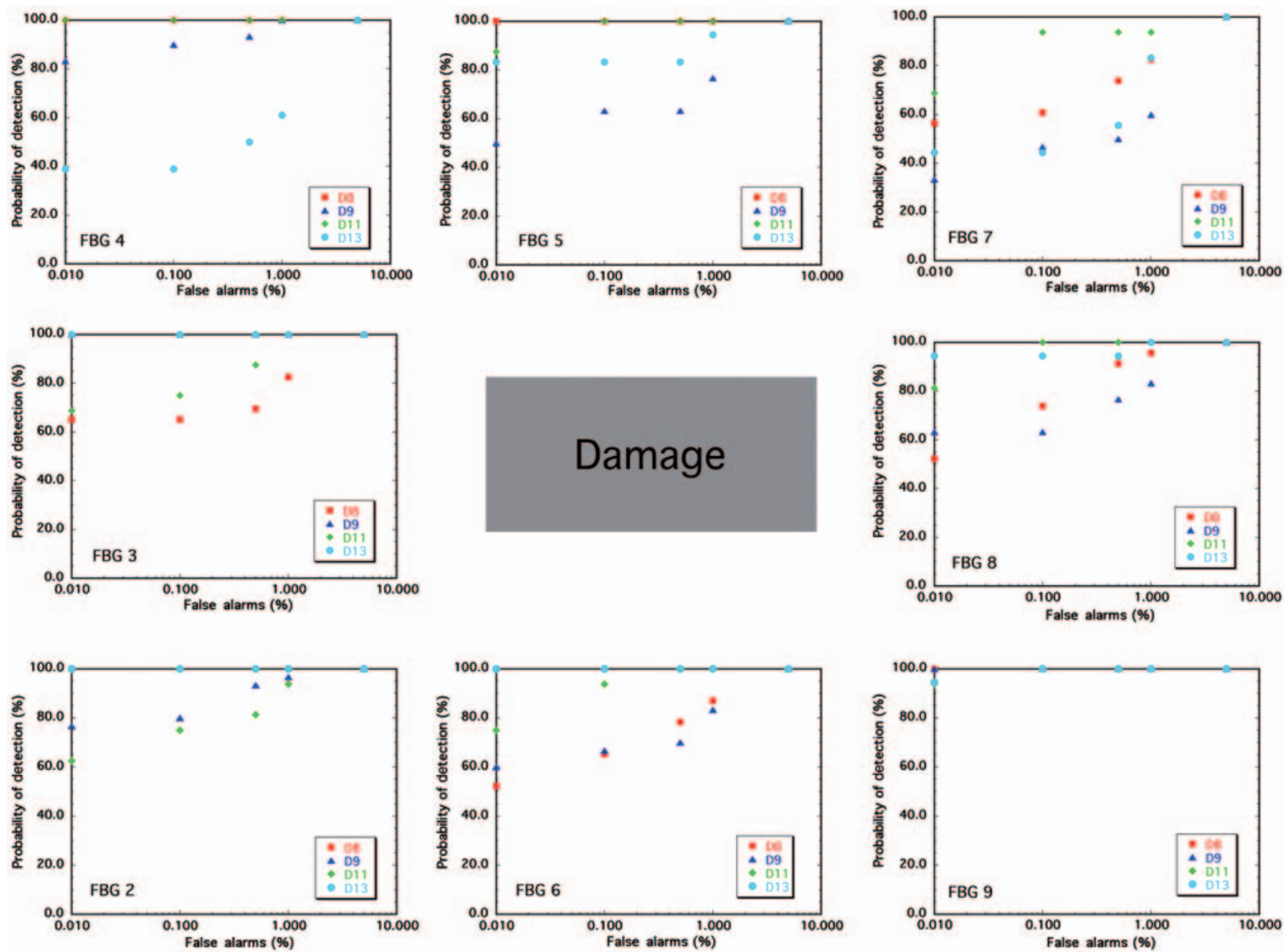

Figure 10. ROC curves for the FBGs as the level of damage increases. The figure is laid out to illustrate the locations of the sensors relative to the damage.

excitation always results in greater POD for a given PFA. In spite of this limitation, several general conclusions can be drawn from these figures. These include the fact that all sensors show greater than $90 \%$ POD at $5 \%$ false alarms. Thus for a 5\% PFA, there is no performance difference between the accelerometers and the FBG strain gages. For PFAs below 5\%, the FBGs have consistently higher PODs for a given PFA than the accelerometers and that the POD difference increases as the PFA drops. Thus, overall the FBGs provide more sensitivity to damage than do the accelerometers. Finally, comparing Figures 10 and 11, there is no clear indication that higher levels of excitation produce higher PODs.

Figures 8 and 9 contain the ROC curves for the three accelerometers with Figure 8 comparing results for the four damage levels and Figure 9 comparing results for damage case 13 when using different levels of excitation. In these two plots, it is clear that the excitation accelerometer (A1) shows slightly higher PODs than the wing tip sensor (A2) and that both of these are noticeably higher than the accelerometer closest to the damage (A3).
Figures 10 and 11 show the ROC curves for the FBGs as we increase the damage or the excitation level, respectively. FBGs 2, 3, 6, 8, and 9 show very high PODs for all false alarm rates at damage level 13 . Note that these sensors either lie between the damage and the wing root (FBG 2, 6, and 9) or along the same span as the damage (FBGs 3 and 8). These sensors also show higher PODs for the other damage cases than FBGs 4, 5, and 7. FBG 9 shows distinctly higher PODs than the other sensors with all values in excess of $90 \%$. Thus, even though FBG 9 measures strain levels that are 30\% smaller than FBG 6, it shows significantly better ROC curve performance.

A major caveat arises because of the hardware changes made between impacts 8 and 9. This change precludes using the data from the previous damage states for direct comparisons. As analyzed, that data showed little if any non-linearity due to the damage. However, the lack of direct comparison means that at this time, we cannot be certain that strain data from the undamaged or lightly damaged wing would show no non-linearity. Thus, a similar set of measurements 

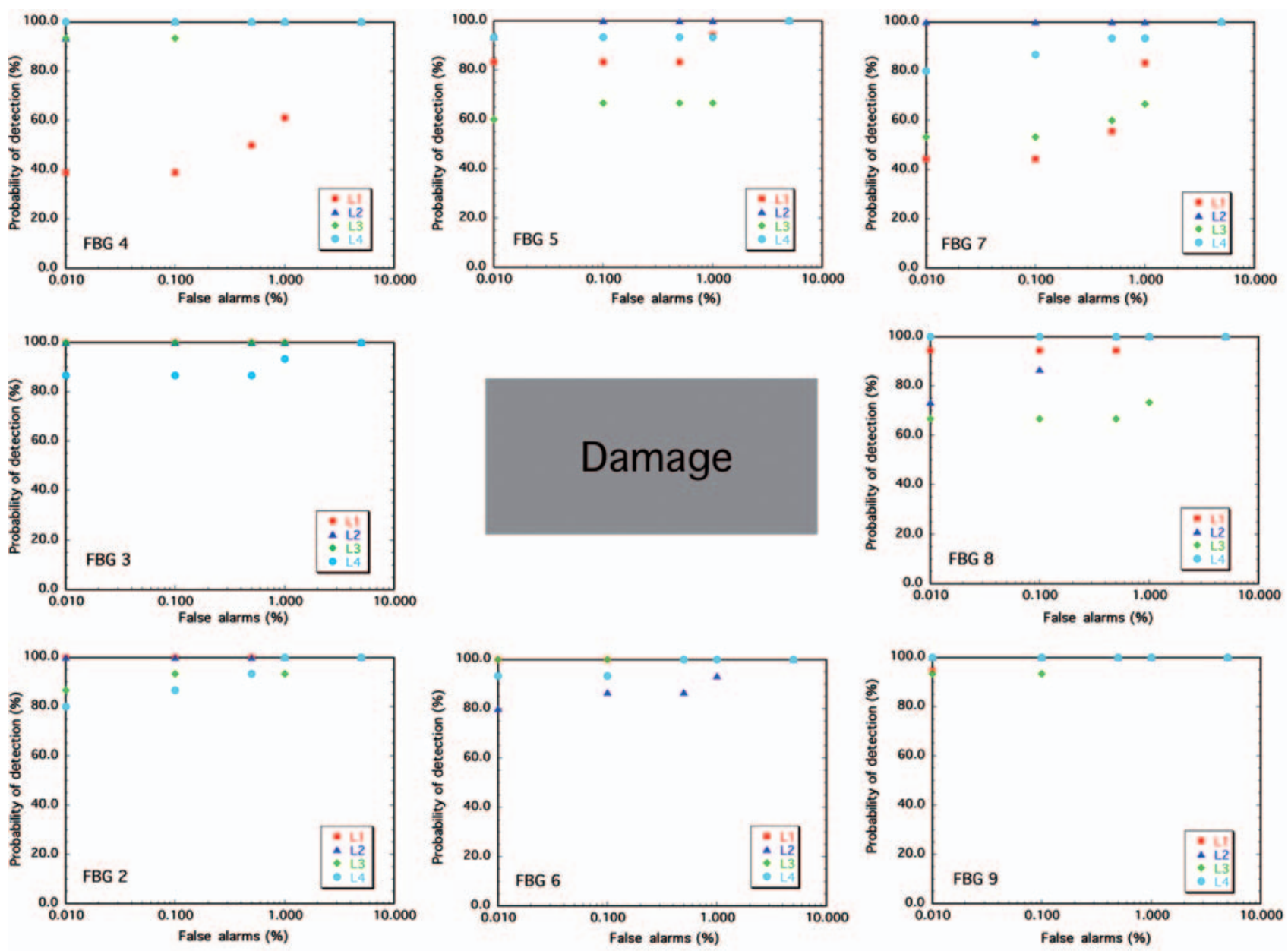

Figure 11. ROC curves for the FBGs following 13 impacts as the level of excitation increases. The figure is laid out to illustrate the locations of the sensors relative to the damage.

starting with an undamaged wing are required to make it clear whether bicoherence can be used as a non-linearity detector for high sensitivity detection of invisible impact damage in foam core composite wing structures such as the one tested here.

\section{CONCLUSIONS}

We have investigated the use of bicoherence as a nonlinearity detector for evaluating low energy impact damage in a foam core composite wing. As a series of non-overlapping impacts $(<7 \mathbf{J})$ produced hidden damage, eight FBGs and three conventional accelerometers recorded the wing's response to Gaussian excitation. The recorded time series were analyzed using bicoherence and assessed as to the presence of nonlinearity by comparing peak heights in the bicoherence results with a threshold calculated from the bicoherence variance. This approach avoids the need for baseline data from the undamaged structure. Variance distribution function thresholds of $95,99,99.5,99.9$, and 99.99 established the probability of false alarms $(5,1,0.5,0.1$, and $0.01 \%$, respectively) as described by Hinich and Wilson (1990). A minimum of 15 measurements spread over 3 days enabled the calculation of ROC curves for all 11 sensors, 4 damage levels, and 4 excitation levels at the highest damage level.

The ROC curve analysis shows that all 11 sensors had at least $90 \%$ POD at 5\% PFA. However, at lower PFAs all FBG strain sensors show consistently higher PODs than the accelerometers. As FBG 5 and accelerometer 3 were nearly co-located, the improved ROC curves indicate that the FBGs are the preferred approach to damage detection in this experiment. The FBG nearest the midchord (F9) and closest to the wing root provided the highest PODs, with $>90 \%$ POD for a $0.01 \%$ probability of false alarms. The other seven FBGs showed similar levels of ROC curve performance. There was inconsistency in the ROC curves with respect to damage level, which requires further investigation to determine if it is inherent at these levels of damage or related to sensor performance. 
Owing to hardware changes made between impacts 8 and 9, we cannot compare the bicoherence results with earlier data from this wing. Thus, we are not able to definitively state that the undamaged wing shows no non-linearity. Further experiments starting with a pristine wing will attempt to minimize response variability due to the FBG demodulation hardware and maintain measurement consistency through a series of impacts.

\section{REFERENCES}

Aktas, E., Seaver, M., Nichols, J.M., Trickey, S.T. and Davis, W.R. 2009. "The Influence of Low Energy Impacts on the Static and Dynamic Response of a Foam Core Composite Wing," Journal of Intelligent Materials Systems and Structures, 20:1351-1361.

Asraf, D.E. and Gustafsson, M.G. 2003. "Optimal Detection of Crack Echo Families in Elastic Solids," Journal of the Acoustical Society of America, 113:2732-2741.

Azarbayejani, M., El-Osery, A.I., Choi, K.K. and Taha, M.M.R. 2008. "A Probabilistic Approach for Optimal Sensor Allocation in Structural Health Monitoring," Smart Materials and Structures, 17, art. \# 055019.

Brockett, P.L., Hinich, M.J. and Patterson, D. 1988. "Bispectral-based Tests for the Detection of Gaussianity and Linearity in Time Series," Journal of the American Statistical Association, 83(403):657-664.

Farrar, C.R., Worden, K., Todd, M.D., Park, G., Nichols, J., Adams, D.E., Bement, M.T. and Farinholt, K. 2007. "Nonlinear System Identification for Damage Detection," Report on a Los Alamos National Laboratory Workshop, \#LA-14353. Available at: http://institute.lanl.gov/ei/annual-workshops/

Frangopol, D.M. and Messervey, T.B. 2008. "Quantifying the Benefits of Smart Technologies in a Life-cycle Context," Advances in Science and Technology, 56:179-188.

Goebel, K.F., Yan, W., Eklund, N.H.W. and Hu, X. 2006. "Defect Classification of Highly Noisy NDE Data Using Classifier Ensembles," In: Inaudi, D., Ecke, W., Culshaw, B., Peters, K.J. and Udd, E. (eds), Proceedings of the SPIE, Smart Structures and Materials, Smart Sensor Monitoring Systems and Applications, Vol. 6167, pp. 460-471.

Hickey, D., Worden, K., Platten, M.F., Wright, J.R. and Cooper, J.E. 2008. "Higher-order Spectra for Identification of Nonlinear Modal Coupling," Mechanical Systems and Signal Processing, doi:10.1016/j.ymssp.2008.10.008.

Hillis, A.J., Nelid, S.A., Drinkwater, B.W. and Wilcox, P.D. 2006. "Global Crack Detection Using Bispectral Analysis," Proceedings of the Royal Society A, 462:1515-1530.

Hinich, M.J. and Wilson, G.R. 1990. "Detection of Non-Gaussian Signals in Non-Gaussian Noise Using the Bispectrum," IEEE Transactions on Acoustics, Speech, and Signal Processing, 38(7):1126-1131.

Hopley, L. and van Schalkwyk, J. 2007. "The Magnificent ROC," Available at: http://www.anaesthetist.com/mnm/stats/roc Findex.htm

Kim, Y.C. and Powers, E.J. 1979. "Digital Bispectral Analysis and its Application to Nonlinear Wave Interaction," IEEE Transaction of Plasma Science, PS-7:120-131.
Lu, Y.H. and Michaels, J.E. 2005. "A Methodology for Structural Health Monitoring with Diffuse Ultrasonic Waves in the Presence of Temperature Variations," Ultrasonics, 9:717-731.

McDonough, R.N. and Whalen, A.D. 1995. Detection of Signals in Noise, Academic Press, New York.

Milanese, A., Marzocca, P., Nichols, J.M., Seaver, M. and Trickey, S.T. 2008. "Modeling and Detection of Joint Loosening Using Output-only Broad-band Vibration Data," Structural Health Monitoring an International Journal, 7:309-328.

Nichols, J.M., Seaver, M., Trickey, S.T., Salvino, L.W. and Pecora, D.L. 2005. "Detecting Impact Damage in Experimental Composite Structures: An Information-theoretic Approach," Smart Materials and Structures, 14:1-11.

Nichols, J.M., Trickey, S.T., Seaver, M., Motley, S.R. and Eisner, E.D. 2007. "Using Ambient Vibrations to Detect Loosening of a Composite-to-metal Bolted Joint in the Presence of Strong Temperature Fluctuations," Journal of Vibrations and Acoustics -transactions of the ASME, 129:710-717.

Nichols, J.M., Trickey, S.T., Seaver, M. and Motley, S.R. 2008. "Using ROC Curves to Assess the Efficacy of Several Detectors of Damage-induced Nonlinearities in a Bolted Composite Structure," Mechanical Systems and Signal Processing, 22:1610-1622.

Okure, M.A.E. and Peshkin, M.A. 1995. "Quantitative Evaluation of Neural Networks for NDE Applications Using the ROC Curve," Review of Progress in Quantitataive Nondestructive Evaluation Parts $A \& B, 14: 2405-2412$.

Olin, B.D. and Meeker, W.Q. 1996. "Applications of Statistical Methods to Nondestructive Evaluation," Technometrics, 38:95-112.

Rivola, A. and White, P.R. 1998. "Bispectral Analysis of the Bilinear Oscillator with Application to the Detection of Fatigue Cracks," Journal of Sound and Vibration, 216:899-910.

Richardson, A.M. and Hodgkiss, W.S. 1994. "Bispectral Analysis of Underwater Acoustic Data," Journal of the Acoustical Society of America, 96:828-837.

Peeters, B. and De Roeck, G. 2001. "One-year Monitoring of the Z24Bridge: Environmental Effects Versus Damage Events," Earthquake Engineering and Structural Dynamics, 30:149-171.

Petrunin, I. and Gelman, L. 2008. "Novel Optimization of Bicoherence Estimation for Fatigue Monitoring," Insight, doi:10.1784/insi.2008.50.3.133.

Shook, B.D., Millwater, H.R., Enright, M.P., Hudak, S.J. and Francis, W.L. 2008. "Simulation of Recurring Automated Inspections on Probability-of-fracture Estimates," Structural Health Monitoring an International Journal, 7:293-307.

Sohn, H., Dzwonczyk, M., Straser, E.G., Kiremidjian, A.S., Law, K.H. and Meng, T. 1999. "An Experimental Study of Temperature Effect on Modal Parameters of the Alamosa Canyon Bridge," Earthquake Engineering and Structural Dynamics, 28:879-897.

Swets, J.A. 1988. "Measuring the Accuracy of Diagnostic Systems," Science, 240:1285-1293.

Todd, M.D., Seaver, M., Bucholtz, F., Nichols, J.M. and Trickey, S.T. 2006. "Modeling and Measurement of Accuracy/Distortion in an Operationally-passive FBG Demodulation Technique," In: Marcus, M.A., Culshaw, B. and Dakin, J.P. (eds), Photonic Sensing Technologies, Proceedings of the SPIE, Vol. 6371, pp. U106-U117. 